\title{
Accompagnement des enseignants en formation initiale. Le point de vue des formés sur la relation tuteur-stagiaire
}

The mentoring of teachers in pre-service training: the trainees' point of view on the tutor-trainee relationship

Acompañamiento de los docentes en formación inicial: el punto de vista de los formados sobre la relación tutor-cursillista

Begleitung der Lehrer in der Erstausbildung: der Gesichtspunkt der Ausg

ebildeten über die Beziehung Mentor-Referendar

Thérèse Perez-Roux

\section{OpenEdition Journals}

Édition électronique

URL : http://journals.openedition.org/rechercheformation/914

DOI : 10.4000/rechercheformation.914

ISSN : 1968-3936

\section{Éditeur}

ENS Éditions

\section{Édition imprimée}

Date de publication : 31 octobre 2007

Pagination : 135-150

ISBN : 978-2-7342-1091-7

ISSN : 0988-1824

\section{Référence électronique}

Thérèse Perez-Roux, «Accompagnement des enseignants en formation initiale. Le point de vue des formés sur la relation tuteur-stagiaire », Recherche et formation [En ligne], 55 | 2007, mis en ligne le 31 octobre 2011, consulté le 19 avril 2019. URL : http://journals.openedition.org/rechercheformation/914 ; DOI : 10.4000/rechercheformation.914

Ce document a été généré automatiquement le 19 avril 2019

(c) Tous droits réservés 


\section{Accompagnement des enseignants en formation initiale. Le point de vue des formés sur la relation tuteur-stagiaire}

The mentoring of teachers in pre-service training: the trainees' point of view on the tutor-trainee relationship Acompañamiento de los docentes en formación inicial: el punto de vista de los formados sobre la relación tutor-cursillista Begleitung der Lehrer in der Erstausbildung: der Gesichtspunkt der Ausg ebildeten über die Beziehung Mentor-Referendar

Thérèse Perez-Roux

\section{Introduction}

1 La formation initiale des enseignants du second degré s'inscrit dans un processus de professionnalisation progressive. Elle s'organise à partir de données institutionnelles soulignant la nécessité pour le professeur d'exercer sa responsabilité à la fois au sein du système éducatif, de l'établissement et de la classe ${ }^{1}$. Ainsi, lors de l'année de formation professionnelle à l'IUFM, les enseignants-stagiaires (PLC2) s'inscrivent dans un dispositif par alternance : enseignants « en responsabilité » dans un établissement d'accueil (collège ou lycée), ils sont aussi stagiaires en formation à l'IUFM. À ce titre, l'institution attend des formés qu'ils articulent progressivement expérience pratique et acquisition de savoirs professionnels ${ }^{2}$, qu'ils soient capables d'analyser leur pratique, dans l'objectif de construire une réflexivité dans et sur l'action (Schön, 1983; Perrenoud, 1994). Ils sont accompagnés dans cette démarche par une équipe de formateurs sur le terrain et à l'IUFM, et par les pairs avec lesquels ils partagent de nombreux moments de formation.

2 La recherche commencée en 2004 s'intéresse aux points d'appui mobilisés durant l'année par les stagiaires, aux tensions et aux adaptations conduisant à l'appropriation 
progressive de compétences professionnelles, envisagées comme un élément majeur dans le processus de construction identitaire (Dubar, 1991 ; Roux-Perez, 2006a).

3 En effet, le moment de l'entrée dans le métier s'avère délicat de ce point de vue, dans la mesure où se rencontrent normes de la formation, représentations initiales des formés et réalité des pratiques. Cette confrontation engage de nombreuses questions.

Tout d'abord, le stagiaire doit préserver un sentiment de continuité vis-à-vis de l'expérience antérieure, tout en se confrontant aux changements inhérents à la complexité du métier, dans un contexte professionnel nouveau pour lui. Par ailleurs, il élabore une « image de soi » en relation - accord, tension, contradiction - avec celles que, selon lui, les autres lui attribuent. Le sentiment de reconnaissance ou de nonreconnaissance d'autrui qui en découle occupe une place essentielle dans la construction identitaire. Une première interrogation apparaît alors: dans quelle mesure les regards croisés sur la pratique - et notamment celui du conseiller pédagogique ou tuteur peuvent aider le stagiaire à se situer et à se définir progressivement comme professionnel?

5 De plus, une autre dimension de la socialisation professionnelle entre en jeu, envisageant le stagiaire en tant que membre "engagé » dans la communauté enseignante (Mead, 1933; Dubar, 1991). En effet, dans son processus d'élaboration, la socialisation professionnelle suppose des identifications à autrui, des rôles à jouer, la découverte de règles explicites ou implicites, la prise en compte d'une organisation scolaire extérieure au stagiaire. À terme, ce processus doit conduire à une reconnaissance du sujet qui, à la fois s'intègre au groupe professionnel, et apprend à jouer son (ses) rôle(s) de manière personnelle et efficace (Hugues, 1955). En fait, pendant la deuxième année de formation à l'IUFM, le processus de socialisation professionnelle s'opère dans des temps, des espaces et des formes d'interactions diversifiés. Il s'agit ici de prendre en compte plus spécifiquement la dynamique de la relation tuteur-stagiaire lors du stage en responsabilité. Durant ce ce stage le conseiller pédagogique a la charge d'accompagner le stagiaire dans la construction des compétences professionnelles attendues par l'institution. Nous nous intéressons à la manière dont s'opèrent les transactions entre ces deux acteurs à l'échelle de l'année de formation. Envisagées comme des actions qui traversent la situation individuelle, les transactions nécessitent délibérations, ajustements, compromis (Dubar, 1992) et conduisent à des formes d'adaptation plus ou moins provisoires, engageant le stagiaire dans sa relation à lui-même, aux autres acteurs et, plus largement, à l'institution. Il convient donc de questionner aussi les effets de contexte inhérents aux affectations et pouvant infléchir de façon radicale le processus de professionnalisation. Le rôle assumé par les tuteurs semble prendre alors une importance capitale pour la construction identitaire du stagiaire. Formateurs légitimés par une compétence professionnelle - voire une forme d'expertise - ils sont invités à aider le stagiaire à diagnostiquer ses difficultés, à en analyser les causes et à choisir les points sur lesquels faire porter prioritairement les efforts. En ce sens, un travail d'accompagnement sur les compétences et les outils pour enseigner est nécessaire (Pelpel, 1996a). Médiateurs dans l'appropriation progressive d'une culture commune, ils veillent à favoriser l'insertion du stagiaire dans l'équipe éducative de l'établissement. Enfin, garants de la formation, ils jouent un rôle dans le dispositif d'évaluation pensé par l'institution. À ce titre, ils s'organisent entre une référence à leur propre pratique d'enseignant, les connaissances du stagiaire et les objectifs de la formation en termes de compétences à acquérir (Faingold, 1996 ; Pelpel, 1996b, 2002). 
Cet ensemble d'éléments rend le conseil délicat dans sa mise en œuvre. Pour le stagiaire, la question de la valeur et de la légitimité de l'opinion du tuteur reste posée, de même que la pertinence et l'opportunité des conseils donnés. Les travaux de Trohel, Chaliès et Saury (2004) mettent en relief différents registres d'activité du stagiaire lors de l'entretien postleçon. Ils pointent un certain nombre de discordances dans la dynamique de l'interaction et des stratégies développées par le "novice»: justification des choix opérés, refus d'entrer dans une discussion contradictoire, exploitation de l'aide réflexive fournie par le tuteur pour tenter d'améliorer la pratique professionnelle, etc.

7 Notre contribution s'intéresse à la relation tuteur-stagiaire dans le processus de professionnalisation. Elle questionne à la fois les effets de contexte institutionnel et humain, l'incidence et la pertinence du regard du conseiller pédagogique dans la construction des compétences professionnelles, les transactions entre des acteurs confrontés à l'épreuve de la pratique, les phénomènes de socialisation à l'échelle de l'année permettant au stagiaire de s'engager dans une relative autonomie professionnelle. Par ailleurs, elle se focalise sur le point de vue du stagiaire dans le dispositif de formation. De fait, elle considère l'inévitable part de subjectivité intégrant représentations sur le métier, idéaux professionnels, chahutés parfois par une pratique professionnelle observée et, en quelque sorte, objectivée par le tuteur.

\section{Repères méthodologiques}

8 L'étude présentée ici prend appui sur une enquête quantitative par questionnaire proposée à l'ensemble des enseignants-stagiaires $(n=277)^{3}$ du second degré des Pays-dela-Loire en novembre 2004. Les réponses issues du questionnaire éclairent les représentations sur le métier les plus partagées par l'ensemble des stagiaires, les difficultés liées à la réalité des pratiques et, par voie de conséquence, les attentes vis-à-vis de la formation en début d'année, notamment au niveau du conseiller pédagogique assurant le suivi du stage en responsabilité (Roux-Perez, 2006c).

Sur ce dernier point, le rôle du tuteur est largement mis en avant par les stagiaires pour progresser dans l'exercice du métier. De fait, les attentes en termes d'accompagnement s'organisent autour de quelques priorités ${ }^{4}$ : que le tuteur " $n$ 'hésite pas à émettre toutes sortes de critiques » (56\%) mais aussi - et sans doute en contrepartie - qu'il «donne des repères » (48\%) et qu'il "guide dans la construction des séances » (42\%). En même temps, son rôle ne se limite pas à cela. Dans le groupe enquêté, 38 \% évoquent d'autres dimensions : "Qu'il rassure et donne confiance » et $32 \%$ «qu'il propose des solutions concrètes ». Certaines réponses soulignent une volonté d'intégration dans le monde des enseignants : le fait que le tuteur "échange avec le stagiaire sur des questions professionnelles" (37\%) ou "qu'il considère le stagiaire comme un collègue à part entière » $(18 \%)$ met en relief cette tendance. Enfin, les formes de non-implication de tuteur conduisant le stagiaire à assumer seul sa tâche sont tout à fait rejetées à ce stade de l'année, voire considérées comme un réel handicap dans la construction d'une professionnalité enseignante.

10 Dans un deuxième temps, 20 stagiaires de différentes disciplines, choisis sur des critères contrastés du point de vue des réponses, ont accepté de s'inscrire dans un dispositif de recherche qualitatif. Le chercheur a conduit trois entretiens semi-directifs (décembre 2004, mars et juin 2005) avec chaque enseignant pour prendre en compte les 
processus de construction de l'identité professionnelle et la manière dont s'opère l'entrée dans le métier.

11 La complémentarité de ces méthodes d'investigation est apparue féconde pour éclairer l'objet de recherche. Suite à une première approche des attentes du groupe professionnel à partir du questionnaire, les entretiens, traités à partir d'une analyse de contenus, offrent la possibilité de saisir chez quelques enseignants débutants ayant vécu des modes d'accompagnement plus ou moins adaptés, la manière dont le processus de professionnalisation a pu s'opérer. A ce niveau, les trois vagues successives de recueil ont constitué un réel intérêt heuristique pour la compréhension:1) des attentes du stagiaire en termes d'accompagnement sur le terrain ; 2) des points d'appui trouvés durant l'année de formation au niveau ou en dehors du tuteur ; 3) de l'évolution professionnelle inscrite entre valeurs, représentations et registres d'action, en lien avec les regards portés sur une pratique plus ou moins assumée.

Nous avons choisi de présenter trois stagiaires qui rendent compte de formes d'accompagnement et de fonctionnement différents. Sophie (mathématiques) et Myriam (lettres) n'ont aucune expérience professionnelle antérieure. Léa (éducation physique et sportive) possède une expérience d'animation dans le cadre associatif; par ailleurs, le cursus universitaire en STAPS 5 intègre une forte dimension de pré-professionnalisation (Roux-Perez, 2006b). Ce corpus permet de suivre les transformations au cours de l'année pour les trois cas étudiés, de manière à repérer dans quelle mesure le mode d'accompagnement tuteur-stagiaire participe du processus de professionnalisation impulsé par l'institution et largement réinterprété par les acteurs.

\section{Résultats}

\section{Balbutiements des premiers jours et attentes envers le tuteur en début d'année}

Pour les trois stagiaires suivis dans l'étude, la prise de contact avec le tuteur s'est opérée en décalage avec des attentes liées aux appréhensions du nouveau statut « d'enseignant en responsabilité ». Dans la salle des professeurs, celui dont chacun attend un certain nombre de repères et de réponses concrètes semble occupé à autre chose, voire manque de temps. De son côté, le stagiaire vit l'urgence du face à face avec les élèves dont il tient à réduire au maximum les zones d'incertitude.

\begin{tabular}{|l|l|l|}
\hline Myriam & Conséquences \\
\hline $\begin{array}{l}\text { "On était un peu noyés : stagiaires, nouveaux arrivants et tout. } \\
\text { Et on a passé la journée au restaurant, c'est vrai que j'étais un } \\
\text { peu désagréablement surprise, par des gens qui après deux mois } \\
\text { de vacances, arrivaient tous crevés. Je les ai suivis au resto. J'en } \\
\text { pouvais plus, ils parlaient de tout sauf des élèves... J'avais peur } \\
\text { parce que je savais que j'avais besoin de soutien cette année. Je } \\
\text { me disais comment passer une heure avec moi pour m'aider s'ils } \\
\text { comptent leurs heures? Et puis finalement, les profs de français } \\
\text { et ma conseillère c'était pas ça du tout, ils ont commencé à } \\
\text { bosser.» }\end{array}$ & $\begin{array}{l}\text { Accompagnement adapté } \\
\text { poure d'un cours } \\
\text { travail collégial }\end{array}$ \\
\hline
\end{tabular}




\begin{tabular}{|c|c|c|}
\hline Léa & $\begin{array}{l}\text { "Le jour de la prérentrée, j'avais envie que mon tuteur vienne } \\
\text { me voir... C'est moi qui ait fait la démarche d'aller me présenter... } \\
\text { et puis chacun a fait son chemin, en fait. Donc ça m'a un peu } \\
\text { dérangée parce qu'on a envie encore qu'on nous prenne un peu } \\
\text { la main. " } \\
\text { "Au début j'avais l'impression qu'il m'échappait en fait, du } \\
\text { genre: “Bon débrouille toi, fais tes armes, on verra bien après". } \\
\text { Donc quelque part je me suis dit : il a raison, l'année prochaine je } \\
\text { serai vouée à moi-même, il faudra que je me débrouille. Mais au } \\
\text { fond de moi... j'étais un petit peu frustrée quand même. Donc au } \\
\text { début ça a été un petit peu dur. Il m'a lâchée, pour moi, dans la } \\
\text { cage aux lions quoi! }\end{array}$ & $\begin{array}{l}\text { Insistance de la stagiaire } \\
\text { pour des informations } \\
\text { permettant de démarrer } \\
\text { les cours dans des } \\
\text { conditions optimales. }\end{array}$ \\
\hline Sophie & $\begin{array}{l}\text { "L'entrée dans la salle des profs ça a été particulier déjà parce } \\
\text { que j'osais pas trop aller vers qui que ce soit, parce que je savais } \\
\text { pas. Ils se retrouvaient eux-mêmes donc c'était pas facile. Et puis } \\
\text { mon tuteur est arrivé à la dernière minute. J'ai eu une très bonne } \\
\text { impression de lui dès le début, et puis... Mais c'est vrai que la } \\
\text { prérentrée ça a été très rapide, parce que moi j'attendais } \\
\text { énormément, j'avais besoin qu'on me guide énormément sur le } \\
\text { premier cours et tout ça. Et il n'avait pas le temps, en fait." }\end{array}$ & $\begin{array}{l}\text { Premières propositions } \\
\text { très décalées avec le } \\
\text { niveau des élèves et } \\
\text { discrédit }\end{array}$ \\
\hline
\end{tabular}

Passées ces premières impressions, se mettent en place des modes de fonctionnement variés au niveau de l'accompagnement sur l'année, plus ou moins adaptés aux contraintes et aux ressources humaines et contextuelles (fonctionnement de l'établissement, caractéristiques des élèves, etc.).

\section{Accompagnement et construction identitaire à travers le regard d'autrui}

\section{Myriam : un accompagnement collaboratif}

Myriam, PLC2 Lettres, a choisi ce métier par goût pour la littérature mais aussi pour sa dimension éducative et pour les possibilités d'ouverture sur le monde que peut offrir cette discipline. Elle effectue son stage dans un établissement de la périphérie nantaise. Elle est suivie par une tutrice expérimentée dans la fonction.

Dans un premier temps, cette formatrice a proposé une présence assidue et s'est inscrite dans un accompagnement basé sur l'écoute et la valorisation du travail de la stagiaire : « Je ne suis pas dans un rapport "expérimenté-bleu" avec elle parce qu'elle peut m'emprunter des cours qu'elle trouve bien. C'est très flatteur pour moi. Elle peut aussi m'en donner pour m'éviter de faire des recherches, parce qu'elle a ça sous la main."

17 Dès le premier entretien, Myriam évoque des qualités chez la conseillère pédagogique rendant compte de certaines «images idéales» de l'enseignant ou du formateur de terrain. Elle reconnaît ainsi à la personne qui l'accompagne, une double compétence :

- dans son rôle de professeur : "C'est quelqu'un de très curieux. C'est vraiment super d'être formée par quelqu'un qui est assez humble et dans le coup pour se former tout le temps. Elle est supercompétente »; 
- dans sa fonction de tutrice : «Vraiment, c'est quelqu'un de très rigoureux, très sérieux... si j'ai besoin d'elle, elle est présente, elle vient et puis elle prend beaucoup de notes et elle me fait un tableau : bien, pas bien, des conseils, etc. Elle est vraiment généreuse de ses idées et de son temps surtout.»

Cette présence attentive pourrait conduire à un envahissement dont Myriam se protège : «Je l'aurais laissé faire, elle serait venue quasiment à tous les cours. Même le jour de la rentrée, elle voulait venir. Je voulais pas quand même!»

En mars, les échanges s'approfondissent, les modes de collaboration s'affinent et les observations croisées participent à la construction progressive des compétences professionnelles. Pourtant, la stagiaire pointe un côté maternant qui gêne parfois la prise d'autonomie, l'obligeant à sortir du seul registre professionnel : "Elle a un côté pas très professionnel, mais très maternel. Ça me fait plaisir parce qu'on s'apprécie beaucoup en dehors du travail, mais en même temps, franchement, des fois j'aimerais qu'elle prenne plus de recul, de distance. Parce que du coup, le côté maternel, j'ai l'impression... de lui devoir quelque chose, tellement elle s'investit. Je me sens un peu obligée d'être une bonne fille (rires). Mais bon, elle est comme ça, je vais pas me plaindre. »

20 Une période de turbulences va apparaître durant le stage de "Pratique accompagnée » (PA) en lycée. Le cadrage très directif imposé par la tutrice de PA, dans lequel Myriam a la sensation d'étouffer, conduit rapidement à un conflit. Le bilan négatif qui en découle et la remise en cause de l'implication de la stagiaire, semble défaire le pacte de confiance avec la tutrice du stage en responsabilité : «J'ai pas aimé tout d'un coup l'espèce de suspicion que ça a créée. Elle se disait : peut-être que je me trompe, c'est peut-être une fumiste. Enfin c'est comme ça que je l'ai senti. "

21 Au final (juin), le retour de la tutrice reste non seulement positif mais souligne les effets d'une construction mutuelle : "Moi, j'ai une conseillère pédagogique super bien à ce niveau-là, qui voit vraiment ça comme un échange. Elle me disait: "Moi je t'apprends des trucs, toi t'es un peu un souffle nouveau, on va travailler ensemble". C'était très valorisant. »

Enfin, une évolution s'est opérée progressivement, sous-tendue par d'autres aspects de la formation et de la validation: "Après, je suis moins allée dans sa classe, elle dans la mienne, et puis finalement, à partir du mois d'avril, j'étais plus dans mon mémoire [qui] m'a pris du temps. J'allais moins la voir, et du coup, on a moins échangé. »

\section{Léa : un accompagnement décalé par rapport aux attentes}

3 Léa, PLC2 EPS, a choisi ce métier par intérêt pour la pratique physique et l'envie de jouer un rôle éducatif auprès des jeunes. Elle effectue son stage dans un établissement rural. Elle est suivie par un tuteur peu expérimenté dans la fonction.

Dans un premier temps, des malentendus sur l'accompagnement et les attentes de la stagiaire ont rendu la relation difficile : "J'ai l'impression que lui il se dit : "si elle a besoin, elle va me le demander", et moi je me dis : c'est quand même à lui de me donner un peu les clefs pour démarrer! Parce que nous en tant que stagiaires on n'ose pas quoi. Donc pour moi le tuteur il faudrait vraiment qu'il soit plus présent au début, quitte à nous demander: "Fais-moi voir ta séance", tout ça, pour que moi je puisse être en confiance et sentir qu’il est là. »

En mars, des compétences autour de la gestion de classe sont construites, reste à adapter davantage les contenus aux caractéristiques des élèves, ce que les regards croisés de différents formateurs vont venir souligner : «Je proposais une situation commune pour tout le 
monde, alors qu'il y avait des élèves qui étaient en difficulté. Moi je le voyais pas, ou si je le voyais, je savais pas quoi faire et il a mis le point dessus. »

À ce niveau, les propositions du tuteur semblent peu opérationnelles. Léa tâtonne, se perd et interpelle son formateur : «J'essaie, mais ça marche pas quoi!». Ce dernier, finit par lui donner quelques pistes: "A chaque séance, essaie de voir quelque chose, de prendre des notes, de voir si cette exigence-là, ils l'ont remplie... Théoriquement, tu as des variables sur tes séances, mais tu les mets pas en application ». La stagiaire cherche, construit, retourne à des données théoriques sur la pédagogie différenciée et tente d'en opérationnaliser certaines : "J'ai essayé de regarder des choses qui étaient acquises... après j'ai réfléchi... Je les ai mis en groupe, et j'ai essayé de proposer donc des situations par niveaux... Et j'étais assez contente ». Cette recherche de différenciation est encouragée par le tuteur qui voit là une réelle transformation de la pratique pédagogique : «Il était content que j'ai essayé de mettre quelque chose en place.»

Dans le même temps, la visite d'un autre formateur vient remettre en cause ces avancées, questionnant les conseils proposés dans l'accompagnement. Ce moment de tension, difficile pour Léa dont la pratique était reconnue jusque-là comme réellement positive, la conduit à formuler de nouvelles demandes, face auxquelles le tuteur ne peut se dérober: "Je lui ai dit: attends, qu'est-ce qui s'est passé? Je comprends pas. En fait, j'avais besoin d'être rassurée.»

Peu à peu, une évolution dans la relation au tuteur est repérée par Léa : «Au début, il était très exigeant et il lâchait pas de leste. Il avait vraiment mis une barrière entre le tuteur et le stagiaire, enfin moi je l'ai vécu comme ça. Et en fait, plus ça va... et plus nos chemins se croisent en fait. Et du coup, plutôt que lui soit dans le jugement, le conseil, maintenant je pense qu'on est à un carrefour où on essaie de construire ensemble. »

Cette construction, évoquée avec pudeur, s'inscrit dans une sorte de co-formation inversée: le tuteur assiste au cours de danse de Léa pour s'y former et tenter de construire des savoirs didactiques et pédagogiques dans cette activité qu'il ne maitrise pas: "Il m’a dit: "écoute, expression corporelle, moi je me verrais pas le faire!", et je lui ai répondu: mais attends... moi, il y a des activités dans lesquelles je me sens moins à l'aise, mais comme elles me sont imposées, c'est pas plus mal, je suis obligée de le faire."

Dans ces conditions, la validation n'inquiète pas cette stagiaire : «On réfléchit ensemble... et ça me montre qu'il me voit plus comme quelqu'un qui doit faire ses preuves, mais comme une collègue... et moi, je me vois déjà l'année prochaine... »

31 Au final, l'intégration dans l'équipe EPS s'est réalisée sans problème, confirmée par une reconnaissance à l'échelle de l'établissement: "Je me suis sentie intégrée assez rapidement dans l'équipe. J'hésite pas à demander des conseils, et puis à l'inverse, quand j'ai des idées je les donne... On m'a vue vraiment comme une collègue assez rapidement. »

\section{Sophie : un accompagnement inadapté}

Sophie, PLC2 Mathématiques et titulaire de l'agrégation, a choisi le métier pour «l'amour des mathématiques et l'envie de faire partager cette passion aux élèves ». Elle effectue son stage dans un établissement classé en ZEP. Elle est suivie par un conseiller pédagogique débutant dans la fonction et nouvellement nommé dans l'établissement.

Dans un premier temps, le travail avec le tuteur s'est organisé autour de la conception et de la planification des cours : activités en commun, suivi de la même progression car l'un 
et l'autre ont une classe de troisième. L'accompagnement porte aussi sur la manière d'introduire les notions au programme de la classe de sixième, point sur lequel Sophie se sent démunie.

Les visites, fréquentes, se centrent essentiellement sur les problèmes de discipline (RouxPerez, 2006a). Les retours sont consignés dans un cahier : ils portent sur « des choses que je fais et qui ne sont pas bonnes ». Par ailleurs, des rencontres avec le professeur principal et le principal adjoint ont eu lieu pour « essayer de régler le problème de l'autorité ».

En mars, les visites se sont espacées, alors que Sophie est plus que jamais débordée par sa classe. Les quelques retours du tuteur sont pris en compte par la stagiaire et réinterprétés "à vue ", ce qui provoque des effets désastreux. Par exemple, le conseil consistant à " passer le plus possible par l'écrit [parce que] ça sert à rien que je leur parle, ils n'écoutent pas » va donner lieu à un " cours sans parole » qui s'avère catastrophique. Pourtant, la stagiaire envisage de poursuivre dans cette voie.

Parallèlement, Sophie prend conscience, lors des visites assurées par d'autres formateurs et lors de l'inspection, de la nécessité d'une réflexion didactique. Des appuis lui sont proposés, non relayés par le tuteur qui «a tendance à ne voir que le côté autorité, parce que lui, il arrive à être bien autoritaire, et du coup il a tendance à regarder que ça». Par voie de conséquence, la question de la validation devient première dans la relation tuteurstagiaire: "Il me renvoie qu'il se pose quand même des questions pour moi, qu'il sait pas, qu'il faut qu'il vienne me voir avec mes sixièmes... Il pense que ça pourrait m'être bénéfique d'avoir une année de plus». Une résistance molle de la stagiaire se met en place face à un tuteur "garant de la formation »: " Moi, j'ai beaucoup de mal à en parler parce que j'ai pas envie de l'influencer, je trouve que c'est à lui de prendre la décision. En même temps, j'ai vraiment pas envie de redoubler."

Lidée d'un contexte de stage très défavorable, voire injuste par rapport aux autres stagiaires, apparait pour la première fois: "J'ai un peu peur qu'en fait il soit tellement exigeant... qu'il me valide pas alors que peut-être qu'avec quelqu'un d'autre je l'aurais été. »

À ce stade de l'année, la confiance dans les compétences du tuteur diminue et la qualité de son suivi est remise en cause par la stagiaire. En effet, le manque de disponibilité pour accompagner une pratique professionnelle problématique est perçu comme un réel handicap par Sophie : «Maintenant, il fait un peu plus le côté didactique, mais c'est vrai qu'on n'a pas beaucoup de temps pour se voir parce qu'il a plein de choses à côté. Il s'occupe de la liaison CM2-sixième...des ordinateurs au collège... En plus, nos emplois du temps correspondent pas trop. Et du coup, c'est moins facile de parler parce que moi j'ai besoin d'être à l'aise avec quelqu'un, de bien le connaitre pour lui parler.» Enfin, des aspects plus personnels sont évoqués par Sophie, soulignant l'impossibilité de prendre en compte des retours du tuteur trop en lien avec une façon singulière d'enseigner: «Et puis on n'a pas du tout la même conception du métier... la même personnalité. On peut pas du tout l'appliquer de la même façon. Ce qui fait qu'il m'apporte pas autant que ce que j'aimerais, mais je sais pas s'il peut m'apporter plus. »

40 En juin, l'année n'est pas validée. Sophie fait le bilan : «Je trouve qu'il a été beaucoup pris par d'autres choses... enfin j'ai eu l'impression de quasiment pas le voir. En fait, c'est surtout d'autres profs qui m'ont apporté. Mais du coup, sur le plan mathématique, c'était pas évident. »

41 La validation semble avoir faussé un travail d'accompagnement adapté au contexte et prenant en compte des aspects à la fois didactiques et pédagogiques. Sophie a vécu cela comme une démission de la part du tuteur: "Je sais que depuis longtemps il estimait qu'il 
valait mieux que je redouble. Moi, j'ai assez mal vécu le fait qu'il m'évalue, et de pas le voir assez souvent. Et en plus, pas de complicité entre nous en fait, on n'a pas construit quelque chose ensemble.»

En même temps, elle se fait le relais d'un tuteur désarmé face à sa mission : «Comme c'était sa première année de conseiller, il a pas été très suivi non plus. Il s'est rendu compte qu'en fait, il aurait fallu qu'il me suive beaucoup plus sur le plan didactique. Mais lui, du coup, il voulait pas trop s'engager parce qu'il savait pas trop ». Renvoyant dos à dos les formateurs qui lui reprochent de ne pas avoir suffisamment analysé sa pratique, et un conseiller pédagogique peu disponible, Sophie relativise au final sa part de responsabilité dans une expérience vécue comme très déstabilisante.

Ces trois cas de stagiaires rendent compte de formes d'accompagnement relativement caractéristiques dans le groupe que nous avons suivi pour l'étude qualitative $(n=20)$. Ainsi, un certain nombre d'interrogations posées en introduction peuvent être éclairées localement et envisagées par ailleurs dans une perspective de généralisation, visant une meilleure compréhension des situations professionnelles liées au conseil pédagogique.

\section{Discussion}

\section{Effets des contextes institutionnels et humains sur la construction de compétences professionnelles}

On constate, au regard de ces trois parcours, de réelles disparités en termes de contexte d'accueil. Si Myriam et Léa enseignent dans un collège relativement calme, Sophie est une des rares stagiaires à effectuer son stage en ZEP. Ainsi, la situation de l'établissement dans des secteurs plus ou moins favorisés socialement, le contexte des classes dans lesquelles sont affectés les enseignants-stagiaires "en responsabilité " jouent donc un rôle non négligeable dans le développement professionnel de ces derniers. Par ailleurs, le type d'accompagnement réalisé par un formateur "de terrain » novice ou expérimenté est prépondérant dans la construction des compétences professionnelles. Cet accompagnement est complexe. En effet, le tuteur peut être dépassé par sa tâche, surtout face à des stagiaires en réelle difficulté. Retours peu critériés, conseils mal hiérarchisés et parfois décalés avec les demandes ou les besoins des formés, peuvent nuire à l'avancée des apprentissages professionnels.

Par ailleurs, les équipes pédagogiques jouent un rôle d'intégration et de régulation important. Léa et Myriam, reconnues professionnellement, peuvent prendre appui sur d'autres collègues de l'établissement pour avancer dans leur réflexion. Sophie se sent entourée, comprise lorsqu'elle exprime ses difficultés de gestion de classe, mais l'aide est surtout d'ordre psychologique. L'équipe cherche à dédramatiser la situation, sans pour autant que la stagiaire parvienne à l'infléchir, notamment d'un point de vue didactique.

Ainsi, plusieurs facteurs, tenant à la fois des acteurs et des contextes traversés, favorisent plus ou moins l'accès à une dynamique de professionnalisation. L'étude pose de fait la question de la formation des conseillers pédagogiques et de leur accompagnement face aux difficultés inhérentes à leur fonction (Pelpel, 1996b). 


\section{Se construire et être reconnu comme professionnel}

47 L'accompagnement engage deux personnes singulières "organisées " à partir d'un système de valeurs-savoirs-pratiques différent. L'enjeu consiste à trouver un terrain d'entente pour travailler ensemble et se donner les moyens de progresser. Ainsi, la rencontre suppose de la part du formé d'accepter le regard d'autrui, d'analyser sa pratique avec l'aide du tuteur, d'accorder une crédibilité aux remarques de ce formateur, de se projeter sur des transformations possibles. Le tuteur a pour rôle d'aider le stagiaire à se repérer dans le flot des impressions laissées par une pratique encore balbutiante. Soumis à des dilemmes tels que "critiquer ou ménager le stagiaire", "former à court terme ou à long terme " (Trohel, Chaliès et Saury, 2004), il est censé évaluer les aspects positifs de la pratique tout en pointant des éléments à améliorer; par ailleurs, il se doit d'accompagner les changements en trouvant la posture la plus adéquate. Dans le cas de Sophie, il semble qu'un malentendu se soit installé, la focalisation du tuteur sur la gestion de classe ayant obstrué l'appui sur des registres plus didactiques indispensables à développer conjointement. Face à des visites espacées vécues comme un abandon, la stagiaire en difficulté a du mal à entendre des conseils qui lui semblent décalés, ou trop loin de ce qu'elle pense être sa vérité pédagogique : «être gentille, aimer tous les élèves, etc. ». Myriam a bénéficié d'une aide efficace doublée de réels échanges professionnels. En ce sens, elle a pu à la fois s'adosser aux remarques constructives de sa tutrice et devenir, en retour, force de proposition pour certaines séquences didactiques. Pour Léa, la rencontre professionnelle s'est réalisée progressivement. Dans un premier temps, la relation au tuteur a été ressentie comme asymétrique, décalée avec ses attentes. Pour améliorer sa pratique, elle a donc sollicité le conseiller pédagogique et s'est appuyée sur d'autres ressources (retours de formateurs, lectures, etc.), mises en synergie pour affiner les questions qui la préoccupaient. En fin de parcours, la présence du tuteur observant des séances de danse pour se former dans une activité qu'il ne maîtrise pas, rend de fait la relation tout à fait symétrique.

Ainsi, le stagiaire construit son identité professionnelle à travers le regard d'autrui. Audelà de celui des élèves, premiers destinataires, le tuteur propose une sorte de miroir rapproché. Au plus près des pratiques effectives, il peut valoriser des éléments de réussite et éclairer telle ou telle zone d'ombre; il peut aussi renvoyer une image déformée ou floue plus difficile à gérer pour le formé. Or, la définition de soi en tant que professionnel suppose une reconnaissance d'autrui fondée sur des retours étayés, argumentés et constructifs légitimant l'entrée progressive du stagiaire dans le monde enseignant.

\section{Processus de socialisation et autonomisation du stagiaire}

Les trois étapes de la socialisation professionnelle repérées par Hugues (1955) s'avèrent bien présentes dans le cheminement des stagiaires. Les ajustements progressifs entre idéaux et normes pratiques supposent, de la part du tuteur, des formes de médiations parfois complexes à saisir et à mettre en œuvre pour permettre une construction identitaire. Celle-ci est traversée par un certain nombre de tensions : d'une part, au niveau biographique, entre continuité et changement; d'autre part, au niveau relationnel, entre soi et autrui. 
50 En effet, l'année de formation invite à une réorganisation des motivations initiales pour le métier élaborées dans le parcours antérieur et la découverte d'un contexte de travail, entre le passage du statut d'étudiant à celui d'enseignant qui nécessite de revisiter des conceptions de l'enseignement souvent fondées sur des images idéales, impossibles à tenir dans l'exercice professionnel. C'est le cas de Sophie qui se positionne avant tout « comme une grande sœur » pour des élèves qui refusent d'entrer dans le jeu scolaire.

51 Conscients de l'importance des compétences pédagogiques et didactiques, traversés par des questions d'éthique professionnelle, les stagiaires sollicitent le plus souvent l'aide du tuteur pour trouver peu à peu des points de repères. La prise de fonction les confronte à des finalités élargies de leur métier et les amène à articuler plusieurs logiques dans la construction de leurs compétences professionnelles. Ces mutations identitaires s'inscrivent dans un processus de socialisation relativement complexe dans lequel le tuteur joue un rôle essentiel, surtout en début d'année. À l'échelle de l'établissement, la relation privilégiée tuteur-stagiaire est relayée progressivement par les autres acteurs (collègues, équipe de direction, parents, etc.).

52 Par exemple, pour Myriam, la reconnaissance de son travail dans l'établissement, le respect de ses choix professionnels constitue un tremplin pour progresser et s'autonomiser. Léa, rapidement reconnue dans une équipe qui l'accepte comme un " professeur à part entière ", se définit à travers des caractéristiques partagées avec la communauté des enseignants et une spécificité liée à ses propres registres de pensée et d'action. Pour la grande majorité des stagiaires suivis dans l'étude, on assiste donc au passage d'un entre-deux identitaire à une identité professionnelle momentanément assumée.

Des différences importantes apparaissent pourtant entre les trois stagiaires suivies dans l'étude. Pour Sophie, la prise d'autonomie reste minime en raison des difficultés importantes rencontrées durant le stage. Les besoins de retours, de conseils sont encore fortement présents en fin d'année et non satisfaits. Pour Myriam, en revanche, après l'appui sur une relation collaborative parfois ressentie comme trop fusionnelle, un malentendu autour du stage de pratique accompagnée favorise la prise de distance. De ce point de vue le mémoire professionnel permet aussi d'investir des aspects du métier revendiqués comme essentiels.

Enfin, pour Léa, la construction de l'autonomie est une perspective qui organise la pratique et le rapport à la formation. Pour se donner les moyens de fonctionner de façon optimale et améliorer son action auprès des élèves, elle s'adosse aux compétences du tuteur qu'elle sollicite quand celui-ci lui semble ne pas entendre ses questionnements professionnels. Cet engagement dans une dynamique de progrès l'amène à envisager l'avenir sereinement à travers une plus grande liberté d'action.

En fait, fondée sur l'entrée « effective » dans le monde professionnel et la reconnaissance d'autrui après validation de la formation, la prise d'autonomie du stagiaire suppose qu'au bout d'un voyage où il a su donner repères et orientations, le tuteur accepte d'entrer dans un processus de distanciation et d'ouverture.

\section{Conclusion}

La manière dont le stagiaire investit les interactions avec le tuteur a inévitablement des effets sur la construction des compétences professionnelles. Si la prise en compte d'un 
nombre conséquent de travaux sur la relation tuteur-stagiaire amène Chaliès et Durand (2000) à discuter l'utilité du tutorat en formation initiale, les études de cas proposées dans cet article soulignent un certain nombre d'éléments plus ou moins favorables pour un accompagnement réussi tant $\mathrm{du}$ point de vue des attendus de l'institution que du développement professionnel des personnes concernées. Une dynamique singulière, faite de décalages, de moments de tensions et d'ajustements conduit le plus souvent à des rencontres réussies, parfois à des rendez-vous manqués. La dimension temporelle révèle la complexité des médiations dans le processus de professionnalisation. Progressivement des dynamiques identitaires apparaissent, laissant place à des formes d'autonomie professionnelle plus ou moins affirmées.

\section{BIBLIOGRAPHIE}

CHALIÈS S., Durand M. (2000). « L'utilité discutée du tutorat en formation initiale des enseignants ", Recherche et formation, $n^{\circ} 35$, p. 145-180.

DUBAR C. $(1991 ; 1996)$. La socialisation : construction des identités sociales et professionnelles, Paris : Armand Colin.

DUBAR C. (1992). «Formes identitaires et socialisation professionnelle », Revue française de sociologie, $\mathrm{n}^{\circ} 4$, p. 505-529.

FAINGOLD N. (1996). « Explicitation des pratiques tutorales et formation de formateurs », Recherche et formation, $\mathrm{n}^{\circ} 22$, p. 81-97.

HUGUES E.C. (1955). “The making of a physician”, Human Organization, III, p. 21-25.

MEAD G.H. (1933). Mind, Self and Society, Chicago : University of Chicago Press.

PAQUAY L. (1996). « Les compétences professionnelles privilégiées dans les stages et en vidéo formation », in L. Paquay, M. Altet, E. Charlier, P. Perrenoud (dir.), Former des enseignants professionnels, Bruxelles : De Boeck, p. 153-179.

PELPEL P. (1996). Guide de la fonction tutorale, Paris : Éditions d'organisation.

PELPEL P. (1996). «Les formateurs de terrain : crise d'identité et évolution du modèle de formation ", Recherche et formation, $n^{\circ} 22$, p. 65-80.

PELPEL P. (2002). « Quelle professionnalisation pour les formateurs de terrain ? ", in M. Altet, L. Paquay, P. Perrenoud, op. cit., p. 176-191.

PERRENOUD P. (1994). La formation des enseignants : entre théorie et pratique, Paris : L'Harmattan.

ROUX-PEREZ T. (2006). Construction des compétences professionnelles chez les enseignants débutants : entre logique de formation, logiques des acteurs et effets de contexte, Colloque « Usages sociaux de la notion de compétences : quels savoirs, quels individus? », Paris (9 mars), www.ensieta.fr/ jecompetence

ROUX-PEREZ T. (2006). « Représentations du métier d'enseignant et rapport à la formation chez les étudiants en STAPS : une identité professionnelle en émergence ", Revue STAPS, n 73, p. 57-69, Bruxelles : De Boeck. 
ROUX-PEREZ T. (2006). Construction identitaire des enseignants en formation initiale : entre représentations partagées et éléments de différenciation dans le rapport au métier, $8^{\mathrm{e}}$ Biennale de l'éducation et de la formation, Lyon (11-14 avril), www.inrp.fr

SCHÖN D.A. (1983). The reflective praticienne, New York : Basic Book.

TROHEL J., CHALIÈS S., SAURY J. (2004). « La dynamique des interactions tuteurs-stagiaires en situation de conseil pédagogique », Revue Savoirs, n 5, p. 121-140.

\section{NOTES}

1. Texte sur la « Mission du professeur exerçant en collège, en lycée d'enseignement général et technologique ou en lycée professionnel », Bulletin Officiel, n² 22, 29 mai 1997.

2. Dans l'IUFM des Pays-de-la-Loire, constituant notre terrain d'étude, le plan de formation (2003-2007) place la pratique professionnelle au cœur de la formation proposée aux enseignantsstagiaires. Cette pratique est entendue comme activité au sein de la classe, de l'établissement, du système éducatif et en liaison avec les partenaires.

3. Le corpus recueilli et exploitable comporte 277 réponses sur la totalité des 343 stagiaires sollicités.

4. Les résultats sont livrés ici sur la base de l'ensemble des réponses (trois choix possibles).

5. Sciences et techniques des activités physiques et sportives.

\section{RÉSUMÉS}

Durant leur formation initiale à l'IUFM, les enseignants-stagiaires du second degré sont invités à s'inscrire dans un dispositif par alternance. Comment investissent-ils les interactions avec le tuteur du stage en responsabilité ? Quels sont les effets de cette relation sur la construction des compétences professionnelles? Pour répondre à ces questions, trois études de cas sont proposées. Elles éclairent décalages, tensions et ajustements dans l'accompagnement tuteurstagiaire. La dimension temporelle de l'étude révèle la complexité des médiations dans le processus de professionnalisation et met en relief des formes d'autonomie professionnelle plus ou moins affirmées.

During their pre-service training at the IUFM, secondary school student teachers are expected to take part in a course combined with work experience. This study deals with the way the students perceive the interactions with the tutor in charge of their work experience and the effects of this relationship on the construction of professional skills. Three case studies are described. They reveal discrepancies, tensions and adaptations in the tutor-trainee support. The temporal dimension of the study shows the complexity of mediation in the professionalization process and brings out some more or less asserted forms of professional autonomy.

Durante su formación inicial en el IUFM, se les invita a los docentes-cursillistas del segundo grado a apuntarse en un dispositivo por alternancia. ¿Cómo invierten las interacciones con el tutor del cursillo en responsabilidad? ¿Cuáles son los efectos de esta relación sobre la construcción de las competencias profesionales? Para responder a estas preguntas se proponen 
tres estudios de caso. Aclaran desfases, tensiones y ajustes en el acompañamiento tutorcursillista. La dimensión temporal del estudio revela la complejidad de las mediaciones en el proceso de profesionalización y pone de manifiesto formas de autonomía profesional más o menos afirmadas.

Während ihrer Erstausbildung an der PH werden die Referendare der Sekundarstufe dazu aufgefordert, einer dualen Ausbildung zu folgen. Wie machen sie sich die Interaktionen mit ihrem Mentor zu eigen? Welche Auswirkungen hat diese Beziehung auf die Erweiterung der Fachkenntnisse? Um diese Fragen zu beantworten, werden drei Fallstudien vorgeschlagen. Sie erklären Diskrepanzen, Spannungen und Anpassungen in der Begleitung Mentor-Referendar. Im Laufe der Zeit deckt die Studie die Komplexität der Schlichtungen im Professionalisierungs prozess auf und betont mehr oder weniger starke Formen beruflicher Autonomie.

\section{AUTEUR}

\section{THÉRÈSE PEREZ-ROUX}

IUFM de Nantes (CREN). 